\title{
Spin flipping a stored polarized proton beam at the IUCF cooler ring
}

\author{
R.A. Phelps \\ University of Michigan \\ Ann Arbor, MI 48109
}

\begin{abstract}
We recently studied the spin flip of a vertically polarized $139 \mathrm{MeV}$ proton beam stored in the IUCF Cooler Ring. We used an rf solenoid to induce a depolarizing resonance in the ring; we flipped the spin by varying the solenoid field's frequency through this resonance. We found a polarization loss after multiple spin flips less than $0.1 \%$ per flip; we also found that this loss increased for very slow frequency changes. This spin flip could reduce systematic errors in stored polarized beam experiments by allowing frequent beam polarization reversals during the experiment.
\end{abstract}

The work reported on here was done in collaboration with D.D. Caussyn, T.J.P. Ellison, S.Y. Lee, T. Rinckel, P. Schwandt, F. Sperisen, E.J. Stephenson, B. von Przewoski of Indiana University, R. Baiod ${ }^{[d]}$, B.B. Blinov ${ }^{[a]}$, C.M. Chu, Don Crandell, Ya. S. Derbenev, W.A. Kaufman, A.D. Krisch, T. Nurushev, T. Roser ${ }^{[b]}$, S. Sund, V.K. Wong ${ }^{[c]}$ of the University of Michigan, E.D. Courant, L.G Ratner of Brookhaven National Laboratory, and C. Ohmori of the University of Tokyo.

Spin flipping a stored polarized proton beam will be essential to reduce systematic errors in any storage ring internal target scattering asymmetry experiment. We have therefore begun a study of techniques for spin flipping a polarized proton beam using an rf solenoid at the Indiana University Cyclotron Facility cooler ring. Similar work was done earlier with polarized electron beams[1].

One uses an rf solenoid to induce an artificial depolarizing resonance in a storage ring at the resonance frequency

$$
f_{r}=f_{c} \times\left(k \pm \nu_{s p}\right)
$$

where $f_{c}$ is the particle circulation frequency, $k$ is an integer, and $\nu_{s p}$, the spin precession frequency measured in units of $f_{c}$, is commonly called the spin tune. When there are no non-vertical magnetic fields present, $\nu_{s p}=G E / m_{p}$, where $G=1.792846$ is the anomalous magnetic moment of the proton. Equation (1) is still valid even if there is a siberian snake present, but then the spin tune has a different energy dependence.

A discussion of the cooler ring, polarimeter and the rf solenoid system used in these studies is in [2]. At every point in the ring, a stable spin direction exists around which the particles spin precesses after one turn around the ring. This "stable spin direction" is normally everywhere vertical. When the rf solenoid is on, the stable spin direction gets a tilt off the vertical which depends on the rf solenoid frequency and field strength. When the rf frequency satisfies equation (1), the stable spin direction is in the horizontal plane. Turning on the rf field at a frequency far to one side of the resonance and ramping the 
frequency through the resonance far to the other side will rotate the stable spin direction from the positive to the negative vertical direction. If this rotation rate is much slower than the spin precession frequency, the spin will follow the stable spin direction and be reversed.

A test of this spin flip technique was performed on a $139 \mathrm{MeV}$ vertically polarized proton beam at the IUCF cooler ring. First, the depolarizing resonance frequency was found by a frequency scan as described in [2] to be $f_{r}=1.8003 \mathrm{MHz}$. Then spin flipping was performed by injecting the polarized beam, turning on the $0.0014 \mathrm{~T}-\mathrm{m}$ rf solenoid field at $f_{i}=f_{r}-1.75 \mathrm{kHz}$ and ramping the frequency to $f_{f}=f_{r}+1.75 \mathrm{kHz}$; we performed this ramp for various ramp times. The data, shown in figure 1 , shows that full spin flip is achieved with ramp times of $20 \mathrm{msec}$ or greater. The curve is a theoretical prediction based on the Froissart-Stora formula[3].

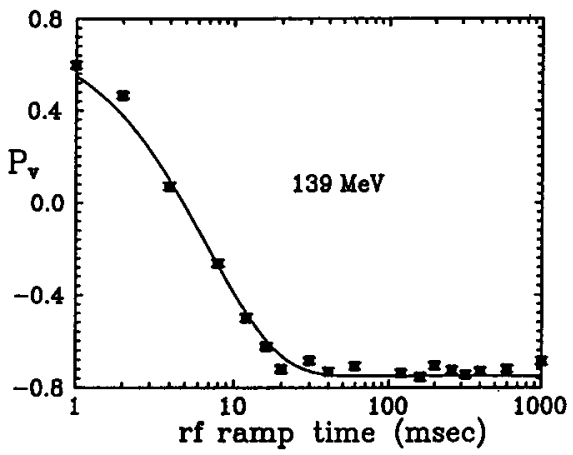

Figure 1: The vertical polarization $P_{v}$ plotted against the rf ramp time through a $3.5 \mathrm{kHz}$ range around the if resonance for a single rf ramp. The curve is the FroissartStora prediction[3].

Even when the spin is completely flipped, there could be some small loss in polarization. We measured this loss for each flip by fixing the rf ramp time at $160 \mathrm{msec}$ and varying the number of ramps before measuring the polarization; the field strength was again $0.0014 \mathrm{~T}-\mathrm{m}$. The data for up to 200 ramps is shown in figure 2 . The curve is $P_{v}=P_{o} p^{n}$, where $n$ is the number of ramps, $P_{o}$ the initial polarization, and $p<1$ is the fraction of polarization that survives each flip. A fit to the data gives $p=0.996$; the polarization loss per flip was about $0.4 \%$. It turns out that there are small polarization losses that increase with the length of the rf ramp. This is demonstrated by fixing the number of ramps to be large to magnify small losses, and increasing the ramp time. Figure 3 shows data for 50 flips and ramp times up to $1 \mathrm{sec}$ per ramp. Note that the polarization increases up to ramp times of $60 \mathrm{msec}$ and then starts down. At $60 \mathrm{msec}$, the polarization loss per flip was better than $0.1 \%$.

This data shows that one can spin-flip a vertically polarized proton beam with very little loss of polarization. This suggests that spin-flip can be used in storage rings to reduce systematic errors in stored polarized proton beam scattering experiments.

This work was supported by grants from the U.S. Department of Energy and the U.S. National Science Foundation. 


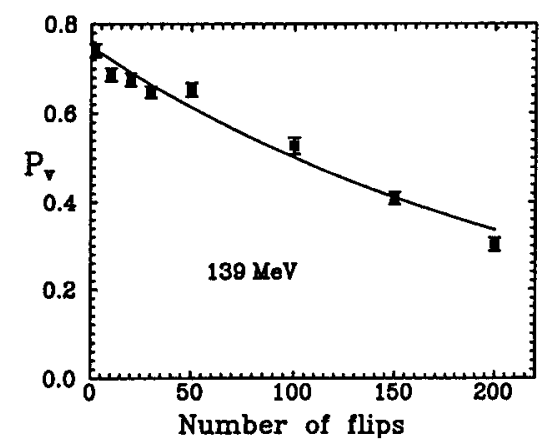

Figure 2: The vertical polarization $P_{v}$ plotted against the number of times the if ramped through a $3.5 \mathrm{kHz}$ range around the rf resonance.

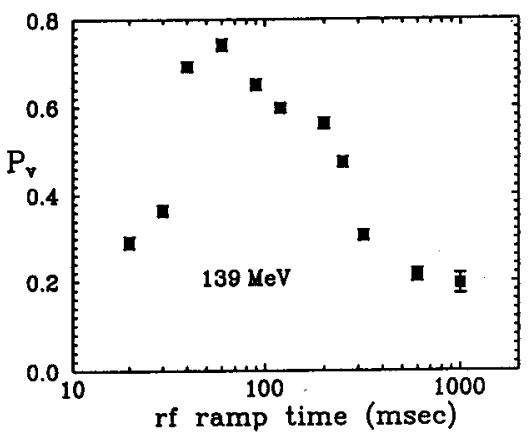

Figure 3: The vertical polarization $P_{v}$ plotted against the rf ramp time through a $3.5 \mathrm{kHz}$ range around the if resonance for one of the 50 ramps performed. Note that the optimum ramp time for efficient spin flip was $60 \mathrm{msec}$.

\section{REFERENCES}

1. A.A. Polunin and Yu.M. Shatunov, Proceedings of Dubna-1981, Polarization Phenomena In High Energy Physics, 308, 1982.

2. A.D. Krisch et al. Phys. Rev. Lett. 63, 1137 (1989),

J.E. Goodwin et al., Phys. Rev. Lett. 64, 2779 (1990),

V.A. Anferov et al., Phys. Rev. A46, R7383 (1992).

3. M. Froissart and R. Stora Nucl. Instrum. Methods 7, 297 (1960).

[a] Also at: Moscow State University, Moscow, Russia.

[b] Present address: Brookhaven National Laboratory, Upton, NY 11973.

[c] Office of the Provost, University of Michigan at Flint.

[d] Present address: Fermilab, Batavia, Dlinois 60510. 